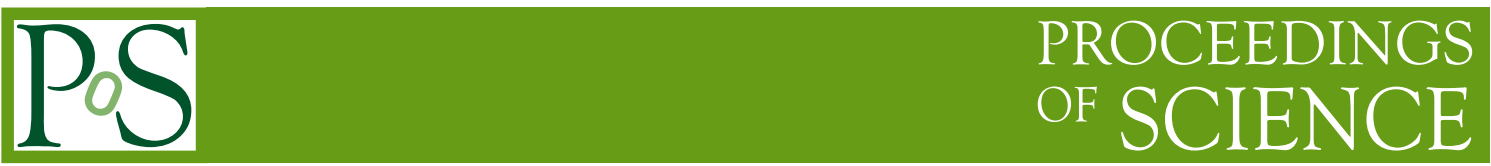

\title{
Vector Boson Scattering: status and prospects
}

\author{
Pietro Govoni* \\ University and INFN Milano-Bicocca \\ E-mail: pietro.govoni@unimib.it
}

\begin{abstract}
The scattering of vector bosons (VBS) happens at the Large Hadron Collider when, in a proton collision, two vector bosons radiate from the interacting quarks and scatter. The VBS process is of great interest because of its intimate relationship with the foundations of the Standard Model of the fundamental interactions, since its unitarity is granted by the EW symmetry breaking. Therefore studying the VBS gives access to the EWSB in an orthogonal way with respect to the Higgs boson physics. Despite being among the rarest processes studied so far, the first results have already been published by the ATLAS and CMS Collaborations, triggering the experimental and theory communities in adding more final states on the measurement side, and pursuing more precision on the theory one. On the long term, the high-luminosity LHC will deliver a dataset of unprecendented size, that will allow to fully exploit the VBS topology, which is nowadays still fully dominated by the statistical uncertainties.
\end{abstract}

Corfu Summer Institute 2018 "School and Workshops on Elementary Particle Physics and Gravity" (CORFU2018)

31 August - 28 September, 2018

Corfu, Greece

${ }^{*}$ Speaker. 


\section{Introduction}

The scattering of vector bosons (VBS) [1] happens at the Large Hadron Collider (LHC) when, in a proton collision, two vector bosons radiate from the incoming quarks and scatter. The decay products of the final state vector bosons, together with the hadronic jets originated by the radiating quarks, determine the signature of the VBS events in the LHC detectors. The process is purely electroweak (EW) at leading order (LO), therefore its production cross-section is among the lowest ever measured by ATLAS and CMS, and only specific features of the VBS events allow for its identification among the various backgrounds. In fact, the two jets, usually called tag jets, carry memory of the initial state momentum and, even if they get deviated enough from the beam axis to enter the sensing volumes of the detectors, are well separated in pseudorapidity. The decay products of the two vector bosons, in turn, are expected to be more central than the two tag jets. Naming $\eta_{1}$ and $\eta_{2}$ the pseudorapidity of the two tag jets (with $\eta_{1}<\eta_{2}$ ), the other fermions in the LO process are then usually required to have $\eta_{1}<\eta<\eta_{2}$, or equivalently $z *<0.5$, where $z *=\left(\eta-0.5\left(\eta_{1}+\eta_{2}\right)\right) /\left(\eta_{2}-\eta_{1}\right)$. The EW nature of the process implies that no colour exchange is expected to happen between the two tag jets, therefore the central region delimited by $\eta_{1}$ and $\eta_{2}$ to be depleted of jets, that would signal hadronic activity typical of a colour flow.

The irreducible background to the VBS signal is due to all the processes that produce a pair of jets and four fermions in the final state, that at leading order happens at $\mathscr{O}\left(\alpha^{6}\right)$ and $\mathscr{O}\left(\alpha^{4} \alpha_{S}^{2}\right)$. Since they show the same final state as the signal, these processes quantum-mechanically interfere with it in the cross-section calculation, therefore in the data analysis the safest way of proceeding is the determination of the fiducial cross section of signal and irreducible background together, after an appropriate set of selections aimed at enhancing the VBS component. Besides the irreducible backgrounds, events where jets get wrongly identified as charged leptons, or charged leptons are not identified because of efficiency or acceptance reasons, or their charge may be mis-reconstructed: these constitute an additional source of contamination of the signal region because of the large cross-section for the production of SM processes accompanied by jets at the LHC. As a consequence, the most sensitive final states studied so far with enough sensitivity to access the SM EW VBS process are characterised by the decay of the vector bosons into electrons or muons.

The VBS process is of great interest at the LHC because of its intimate relationship with the foundations of the Standard Model (SM) of the fundamental interactions. In fact, the EW symmetry breaking (EWSB), besides giving mass to vector bosons and predicting the existence of the Higgs boson, grants the unitarity of VBS. In absence of the Higgs boson, namely, the VBS cross-section would diverge at high energy and only a precise cancellation of oppositely diverging effects tames this behaviour [2]. The observation of the VBS process is therefore complementary to the Higgs boson discovery [3], testing the EWSB mechanism at a very different evergy regime. Since the Higgs boson production cross-section measurement is subject to non-negligible uncertainties, the VBS process offers also additional handles to identify any possibile deviations of Nature from the SM predictions. For example, even small variations of the Higgs boson couplings to other particles would jeopardise the delicate balancing of the single contributions in the VBS crosssection calculation, rendering it divergent $[4,5]$. The systematic studies of these effects is usually performed in the frame of the Effective Field Theory (EFT), that, starting from the SM as an effective low-energy approximation of a more comprehensive model parametrises any deviations 
from the SM Lagrangian with dimension-eight operators [6], assuming that other measurements would effectively constrain dimension-six ones. Nevertheless, recent studies show that the VBS final state has impact also on dimension-six ones [7], and with a complete set of operators [8] fully implemented as UFO model usable in modern event generators [9], the time has come to exploit the LHC dataset in the VBS phase space to contribute to the overall dimension-six constraints, that would also be the natural thing to check in case any deviations from the SM expectations should arise in future measurements. In this framework, these first two categories of higher order terms modify the SM Lagrangian as follows:

$$
\mathscr{L}_{E F T}=\mathscr{L}_{S M}+\sum_{i=1}^{N_{d 6}} \frac{c_{i}}{\Lambda^{2}} \mathscr{O}_{i}^{(6)}+\sum_{j=1}^{N_{d 8}} \frac{c_{j}}{\Lambda^{4}} \mathscr{O}_{j}^{(8)},
$$

where $\mathscr{O}$ represent higher order operators, $\Lambda$ the energy scale below which the EFT approximation is valid, and $c_{i}$ the coefficients tuning the importance of each operator in the EFT Lagrangian. The impact of the EFT terms parameterising effects beyond the SM (BSM) is expected to be noticeable at the highest energies accessible at the LHC, as they are the ones closest to the regime where new physics would become relevant. Experimentally, this implies that the sensitivity to BSM effects is connected mostly to the number of events collected in the high-energy tails of the spectra. In this corner of the phase space backgrounds often fade with respect to their intensity in the low-energy regime where the SM VBS signal is expected, therefore final states which are not significative for the SM VBS search might become very powerful in the EFT paradigm. To effectively investigate the high-energy domain, specific algorithms may be deployed to properly reconsctruct and identify the final state particles, as is the case for hadronically-decaying vector bosons originating single large jets in the detector, or highly-energetic charged leptons.

In the following sections an overview of the most recent existing VBS measurements performed by the ATLAS and CMS Collaborations is presented, followed by a summary of the most recent theory developmements in the field. The last paragraph will show prospect studies carried on by the ATLAS and CMS Collaborations, and will be followed by the conclusions to this document.

\section{Experimental results}

The most relevant result in the VBS analysis in the recent years is the observation of the samesign W boson scattering, obtained by both ATLAS and CMS Collaborations [10, 11, 12, 13] with about $36 \mathrm{fb}^{-1}$ of integrated luminosity. The main peculiarity of this channel is the charge equality of the two final state vector bosons: very few other processes produce the signature of two samecharge leptons in the detector, therefore this channel, despite having a cross-section of the order of a few femtobarns, exhibits an excellent signal-over-noise ratio.

The main selections applied in this case by the ATLAS and CMS Collaborations are shown in Table 1. The first two selections correspond to minimal kinematic requests on the final state topology. The following three lines report typical VBS selections: the two tag jets are well separated along the $\eta$ coordinate and, because of the large fraction of proton energy they carry, their system exhibits a large invariant mass; the charged leptons are required be central in the event with respect to the tag jets. The remaining ones suppress backgrounds by discarding events characterised by the presence of b-tagged jets, additional charged leptons besides two same-sign ones, or dilepton 


\begin{tabular}{|l|c|c|}
\hline & ATLAS & CMS \\
\hline$E_{T}^{m i s s}>[\mathrm{GeV}]$ & 30 & 40 \\
$p_{T}^{j}>[\mathrm{GeV}]$ & $65-35$ & $30-30$ \\
$m_{j j}>[\mathrm{GeV}]$ & 500 & 500 \\
$\Delta \eta_{j j}>$ & 2.0 & 2.5 \\
$\max \left(z *_{\ell}\right)<$ & - & 0.75 \\
$m_{\ell \ell}>[\mathrm{GeV}]$ & 20 & 20 \\
$\left|m_{e e}-m_{Z}\right|>[\mathrm{GeV}]$ & 15 & 15 \\
no additional charged leptons & applied & applied \\
b-jets veto & applied & applied \\
\hline
\end{tabular}

Table 1: Main selections operated by the ATLAS and CMS Collaboration in the seach for the same-sign WW scattering.

resonances. The distribution of two significative variables after the event selections are shown in Figure 1: with the data collected by the ATLAS Experiment, on the left, the spectrum of $m_{j j}$ is shown, while for the events collected by the CMS Collaboraion, on the right, the spectrum of $m_{\ell \ell}$ is reported. The data counts for each bin, in black dots, are overlapped to the sample composition estimates, where in both cases the signal is clearly visible above the various backgrounds.
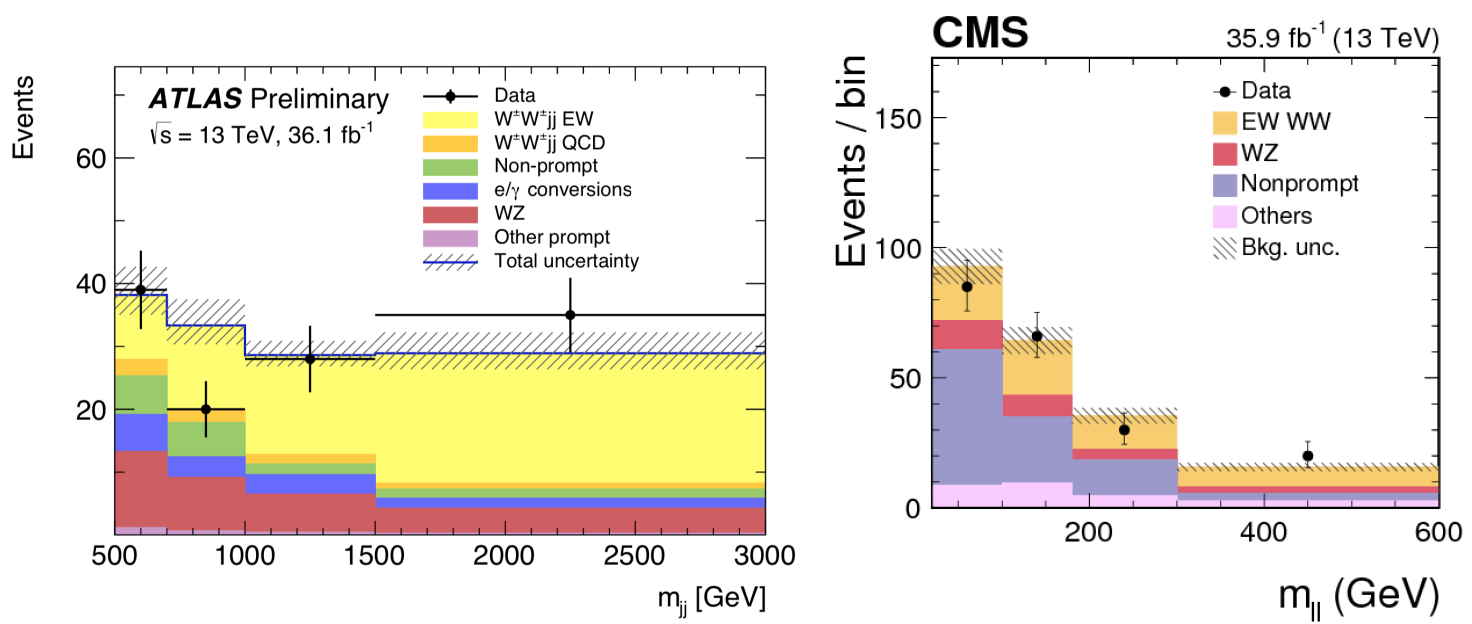

Figure 1: Distribution of two significative variables after the event selections, for the same-sign WW scattering. For the data collected by the ATLAS Experiment, on the left, the spectrum of $m_{j j}$ is shown, while for the events collected by the CMS Collaboraion, on the right, the spectrum of $m_{\ell \ell}$ is reported.

For both measurements, the observed signficance exceeds the 5 standard deviations (6.9 s.d. for ATLAS, 5.5 s.d. for CMS), while the expected one is a bit larger in the case of the CMS data analysis with respect to the ATLAS one (4.6 s.d. for ATLAS, 5.7 s.d. for CMS). The two Collaborations published also a fiducial cross-section associated to the VBS process in question. In the ATLAS case, the value is $\sigma_{\text {fid }}=3.83 \pm 0.66$ (stat) \pm 0.35 (syst) fb, while in the CMS measurement reads: $\sigma_{\text {fid }}=2.950 .49$ (stat) 0.23 (syst) fb, and the research groups are now in the process of comparing the two studies step-by-step. Both results are statistically dominated and exploit only 
a fraction of the total number of collisions delivered by the LHC, therefore significative improvements are expected already by studying the whole dataset collected by the two experiments.

The second signature in terms of sensitivity to the SM VBS process is characterised by the presence of a WZ pair in the final state, with both vector bosons decaying leptonically. In this case, the analysis strategies adopted by the two Collaborations differ significantly from one another [14, 15, 16]: while the ATLAS one opted for a multi-variate classifier aimed at reducing the background contamination, the CMS Collaboration implemented a conservative approach based on rectangular selections. The expected significances of the analyses are similar (3.3 s.d. for ATLAS, 2.7 s.d. for CMS), and the observed one are quite different: an overfluctuation brings the result above the 5 s.d. threshold for the ATLAS case, while an underfluctuation penalises the CMS one (5.6 s.d. for ATLAS, 1.9 s.d. for CMS). In this channel, the backgrounds are larger with respect to the same-sign WW one, reducing the expected sensitivity. The attentive reader will also notice that the WZ channel may originate background events in the same-sign $\mathrm{W}$ one, in case of inefficiencies or mistakes in the event reconstruction. Therefore, once more events will become available, the data analysis may develop in the direction of measuring the two processes jointly.

The third fully leptonic channel investigated so far is the final state where two $\mathrm{Z}$ bosons decay into electrons or muons. Despite the low cross section (at the order of $0.5 \mathrm{fb}$ ), the presence of four charged leptons allows for the reconstruction of a very clean final state, to the level that the CMS Collaboration was able to observe the VBS signal with 2.7 s.d. with respect to the background-only hypothesis [17], making use of a boosted decision tree trained to segregate the VBS signal from the rest of the processes.

In all these cases, no significative deviations from the SM expectations have been observed, even at the highest energies within reach, therefore limits have been set on the presence of dimensioneight operators modifying the SM Lagrangian, under the assumption that dimension-six ones are not present. In these studies, besides fully-leptonic final states also the semi-leptonic ones become relevant, as they profit of the larger statistics due to the vector boson hadronic branching ratio. Both ATLAS and CMS $[18,19]$ studied these topologies, where the signal competes primarily with a background due to the production of single leptonically-decaying vector bosons accompanied with jets. Their importance is estimated with data-driven techniques, devised by the analysis teams to cope with the uncertainties in the Monte Carlo simulation, which are due to the limited knowledge of the process (for example, it is practically known at LO only) and the difficulty of producing simulated samples with negligible statistical uncertainty. Figure 2 shows, on the left, an example of the impact of a dimension-eight operator on the invariant mass spectrum of the $\mathrm{ZV}$ system, where the $\mathrm{Z}$ boson decays leptonically and the $\mathrm{V}$ one hadronically (this includes both $\mathrm{W}$ and $\mathrm{Z}$ bosons, since the experimental separation between the two is not currently feasible at the LHC, due to the limited energy resolution in reconstructing jets), from a CMS study. On the right, the result of a two-dimesional fit of BSM operators from an ATLAS study is shown, in terms of confidence regions obtained from the measurement. The power of the semi-leptonic results, displaied in black, is clearly visible when compared to the ones of the fully-leptonic analyses.

\section{Theory developments}

With the dataset collected by ATLAS and CMS until now still to be fully exploited and much 

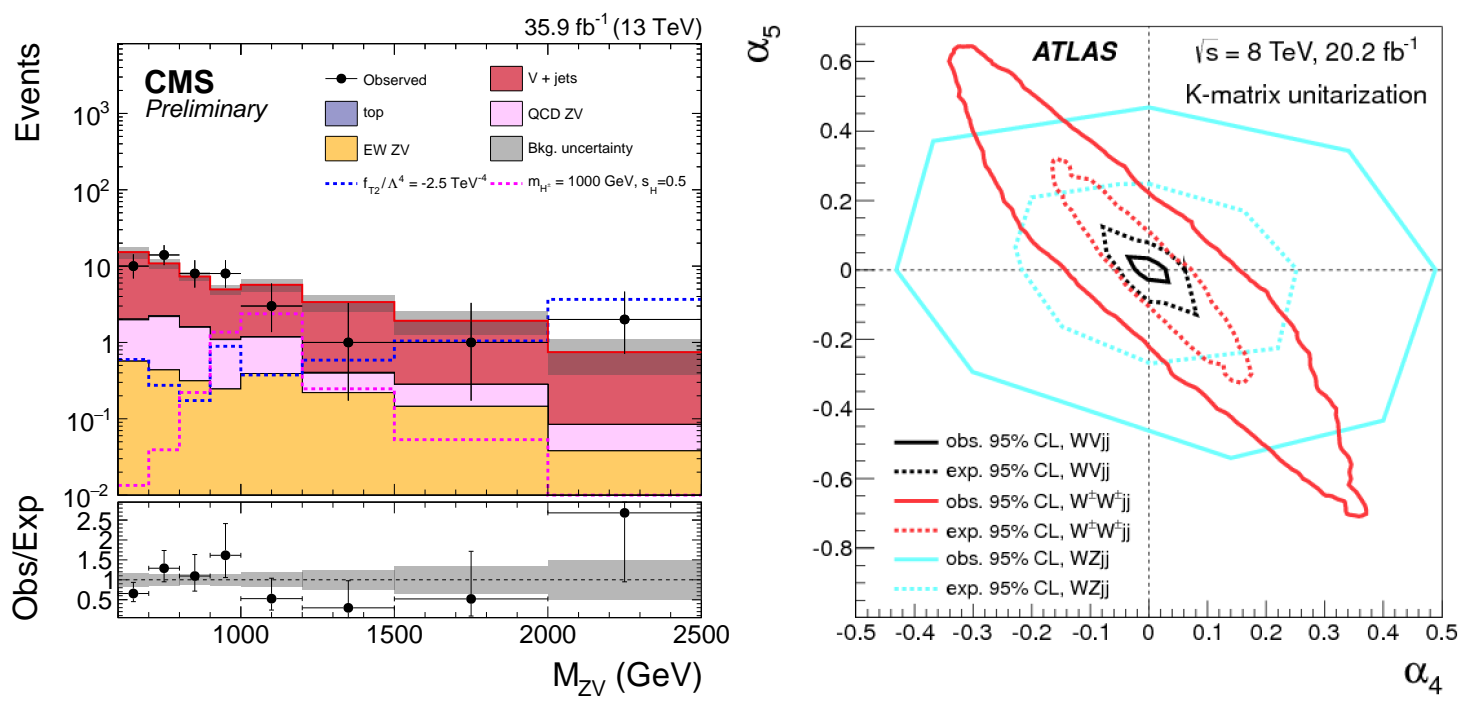

Figure 2: On the left, an example of the impact of a dimension-eight operator on the invariant mass spectrum of the ZV system, where the Z boson decays leptonically and the V one hadronically, from a CMS study. On the right, the result of a 2-dimesional fit of BSM operators from an ATLAS study is shown, in terms of confidence regions obtained from the measurement.

more event to come in the following years, the experimental precision that will be attained needs to be matched by a theoretical understanding of the VBS process with corresponding accuracy. The VBS process is particularly challenging, since calculations are complex because of the large number of diagrams involved, and the EW higher order corrections may be comparable to the QCD ones. The large number of particles in the final state, in fact, can be produced in a multitude of ways also for the simplest cases, where even the hypothesis that two vector bosons scatter is not granted when calculating the full interaction already at LO in $\mathscr{O}\left(\alpha^{6}\right)$.

The first calculation of the complete set of NLO corrections to the VBS, in both the EW and QCD part, have been performed for the same-sign WW scattering [20] and for the WZ one [21] in the case of fully-leptonic final states, and show that large EW corrections are an intrinsic feature of VBS at the LHC, as they may reach an impact larger than $10 \%$ on fiducual cross sections and relevant effects on differential distributions, as exemplified in Figure 3. On the left panel, the differential cross-section for the invariant mass of the di-lepton system, in the case of $e^{+} \mu^{+}$, is shown for the LO calculation and the NLO one, where both QCD and EW terms are considered. Around central values, the impact of scale variations is shown as well for each prediction.

While these calculations have been performed with the full set of diagrams contributing to the same-sign WW or WZ final states and practically demonstrated one techqniue to get the full NLO corrections to VBS, the LHC data analysis addresses all accessible final states and is performed independently by the experimental collaborations. Novel developments in the field therefore are also meant to support the measurements, by providing a consistent picture of the VBS simulation with a comparative study of the existing calculations and Monte Carlo programs [22]. For example Figure 3, on the right panel, shows the differential distribution in the $z *$ variable of the third-hardest jet from predictions matched to parton showers, at QCD NLO accuracy, where significative differ- 

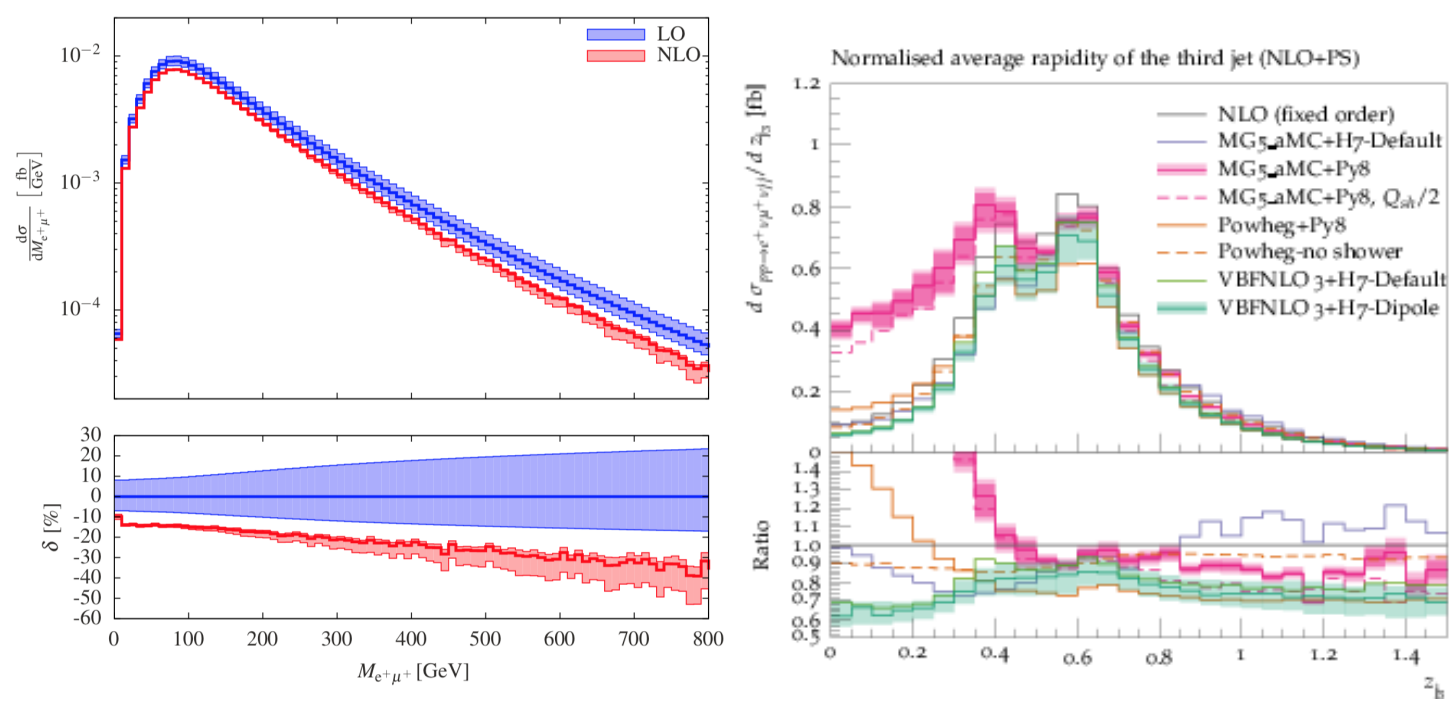

Figure 3: On the left, the differential cross-section for the invariant mass of the di-lepton system, in the case of $e^{+} \mu^{+}$, is shown for the LO calculation and the NLO one, where both QCD and EW terms are considered. Besides central values, the impact of scale variations is shown as well for each prediction. On the right, the differential distribution in the $z *$ variable of the third-hardest jet from predictions matched to parton showers, at QCD NLO accuracy.

ences are present among different tools, due to the limited precision in the perturbative description of observables connected to particles present in the event only at higher orders. This distribution, in particular, is tightly connected to the understanding of the effects of any hadronic activity vetoes that may be used to separate a signal from its backgrounds.

\section{Future prospects}

Because of their low production cross-section, only a few VBS processes have been measured so far, and the existing results give inclusive informations only. As the collected statistics will significantly increase in the future [23], ATLAS and CMS are reoptimising particle reconstruction strategies $[24,25]$ in view of the high-luminosity phase of the LHC in particular. The whole chain of the data analysis will be affected, since in this new challenging environments the ATLAS and CMS detectors themselves will undergo upgrades, meant to cope with the increased pile-up and the harsher radiation environment. This in turn will reflect on the data analysis strategies, from the triggering procedures to the final state particles reconstruction and the accessible phase space for the analysis. This will allow to pursue even more ambitious targets, such as the measurement of the longitudinal component of the VBS, which is the one actually sensitive to the EWSB. Preliminary feasibility studies have been performed already by the CMS Collaboration [5], showing that with our current understanding its discovery remains a challenging objective, requiring improvements on the experimental and theory sides. For example for the latter, activities are already ongoing, in order to understand how to effectively isolate the EWSB effects in the longitudinal VBS in a sound manner from the point of view of the theory predictions [26], ensuring the correctness of any 
approximations performed in the analysis, for example, identifying the most sensitive variables to be tackled to maximise the sensitivity to BSM effects.

\section{Conclusions}

The scattering of vector bosons provides access to the EWSB in an orthogonal way with respect to the Higgs boson physics, as it targets a very different energy regime and one crucial aspect of the SM, the unitarity of the VBS. Despite being among the rarest processes studied so far, the first results have already been published by the ATLAS and CMS Collaborations, triggering the experimental and theory communities in adding more final states on the measurement side, and pursuing more precision on the theory side. On the long term, the high-luminosity LHC will deliver a dataset of unprecendented size, that will allow to fully exploit the VBS topology, which is nowadays still fully dominated by the statistical uncertainties.

\section{References}

[1] C. F. Anders et al., "Vector boson scattering: Recent experimental and theory developments," Rev. Phys. 3 (2018) 44 doi:10.1016/j.revip.2018.11.001 [arXiv:1801.04203 [hep-ph]].

[2] A. Alboteanu, W. Kilian and J. Reuter, "Resonances and Unitarity in Weak Boson Scattering at the LHC," JHEP 0811 (2008) 010 doi:10.1088/1126-6708/2008/11/010 [arXiv:0806.4145 [hep-ph]].

[3] G. Aad et al. [ATLAS and CMS Collaborations], "Combined Measurement of the Higgs Boson Mass in $p p$ Collisions at $\sqrt{s}=7$ and $8 \mathrm{TeV}$ with the ATLAS and CMS Experiments," Phys. Rev. Lett. 114 (2015) 191803 doi:10.1103/PhysRevLett.114.191803 [arXiv:1503.07589 [hep-ex]].

[4] K. Cheung, C. W. Chiang and T. C. Yuan, "Partially Strong WW Scattering," Phys. Rev. D 78 (2008) 051701 doi:10.1103/PhysRevD.78.051701 [arXiv:0803.2661 [hep-ph]].

[5] CMS Collaboration, "Prospects for the study of vector boson scattering in same sign WW and WZ interactions at the HL-LHC with the upgraded CMS detector," CMS Physics Analysis Summary CMS-PAS-SMP-14-008, [https://cds.cern.ch/record/2220831].

[6] O. J. P. Eboli, M. C. Gonzalez-Garcia and J. K. Mizukoshi, " $p p \rightarrow j j e^{ \pm} m u^{ \pm} v v$ and $p p \rightarrow j j \pm m u \mp v v$ at $\mathscr{O}\left(\alpha_{e m}^{6}\right)$ and $\mathscr{O}\left(\alpha_{e m}^{4} \alpha_{s}^{2}\right)$ for the study of the quartic electroweak gauge boson vertex at CERN LHC," Phys. Rev. D 74 (2006) 073005 doi:10.1103/PhysRevD.74.073005 [hep-ph/0606118].

[7] R. Gomez-Ambrosio, "Studies of Dimension-Six EFT effects in Vector Boson Scattering," arXiv: 1809.04189 [hep-ph].

[8] B. Grzadkowski, M. Iskrzynski, M. Misiak and J. Rosiek, "Dimension-Six Terms in the Standard Model Lagrangian,” JHEP 1010 (2010) 085 doi:10.1007/JHEP10(2010)085 [arXiv:1008.4884 [hep-ph]].

[9] I. Brivio, Y. Jiang and M. Trott, “The SMEFTsim package, theory and tools," JHEP 1712 (2017) 070 [arXiv:1709.06492 [hep-ph]].

[10] A. M. Sirunyan et al. [CMS Collaboration], "Observation of electroweak production of same-sign W boson pairs in the two jet and two same-sign lepton final state in proton-proton collisions at $\sqrt{s}=13$ TeV,’ Phys. Rev. Lett. 120 (2018) no.8, 081801 doi:10.1103/PhysRevLett.120.081801 [arXiv:1709.05822 [hep-ex]]. 
[11] V. Khachatryan et al. [CMS Collaboration], "Study of vector boson scattering and search for new physics in events with two same-sign leptons and two jets," Phys. Rev. Lett. 114 (2015) no.5, 051801 doi:10.1103/PhysRevLett.114.051801 [arXiv:1410.6315 [hep-ex]].

[12] ATLAS collaboration, "Observation of electroweak production of a same-sign $W$ boson pair in association with two jets in $p p$ collisions at $\sqrt{s}=13 \mathrm{TeV}$ with the ATLAS detector," ATLAS-CONF-2018-030.

[13] M. Aaboud et al. [ATLAS Collaboration], "Measurement of $W^{ \pm} W^{ \pm}$vector-boson scattering and limits on anomalous quartic gauge couplings with the ATLAS detector," Phys. Rev. D 96 (2017) no.1, 012007 doi:10.1103/PhysRevD.96.012007 [arXiv:1611.02428 [hep-ex]].

[14] CMS Collaboration, "Measurement of electroweak WZ production and search for new physics in pp collisions at sqrt(s) = 13 TeV," CMS-PAS-SMP-18-001.

[15] ATLAS collaboration, "Observation of electroweak $W^{ \pm} Z$ boson pair production in association with two jets in pp collisions at $\sqrt{s}=13 \mathrm{TeV}$ with the ATLAS Detector," ATLAS-CONF-2018-033.

[16] G. Aad et al. [ATLAS Collaboration], "Measurements of $W^{ \pm} Z$ production cross sections in $p p$ collisions at $\sqrt{s}=8 \mathrm{TeV}$ with the ATLAS detector and limits on anomalous gauge boson self-couplings,” Phys. Rev. D 93 (2016) no.9, 092004 doi:10.1103/PhysRevD.93.092004 [arXiv:1603.02151 [hep-ex]].

[17] A. M. Sirunyan et al. [CMS Collaboration], "Measurement of vector boson scattering and constraints on anomalous quartic couplings from events with four leptons and two jets in proton-proton collisions at $\sqrt{s}=13$ TeV,' Phys. Lett. B 774 (2017) 682 doi:10.1016/j.physletb.2017.10.020 [arXiv:1708.02812 [hep-ex]].

[18] M. Aaboud et al. [ATLAS Collaboration], "Search for anomalous electroweak production of $W W / W Z$ in association with a high-mass dijet system in $p p$ collisions at $\sqrt{s}=8 \mathrm{TeV}$ with the ATLAS detector,” Phys. Rev. D 95 (2017) no.3, 032001 doi:10.1103/PhysRevD.95.032001 [arXiv:1609.05122 [hep-ex]].

[19] CMS Collaboration, "Search for anomalous electroweak production of WW/WZ/ZZ boson pairs in association with two jets in proton-proton collisions at 13 TeV," CMS-PAS-SMP-18-006.

[20] B. Biedermann, A. Denner and M. Pellen, "Complete NLO corrections to $\mathrm{W}^{+} \mathrm{W}^{+}$scattering and its irreducible background at the LHC," JHEP 1710 (2017) 124 doi:10.1007/JHEP10(2017)124 [arXiv:1708.00268 [hep-ph]].

[21] A. Denner, S. Dittmaier, P. Maierhöfer, M. Pellen and C. Schwan, "QCD and electroweak corrections to WZ scattering at the LHC," arXiv:1904.00882 [hep-ph].

[22] A. Ballestrero et al., "Precise predictions for same-sign W-boson scattering at the LHC," Eur. Phys. J. C 78 (2018) no.8, 671 doi:10.1140/epjc/s10052-018-6136-y [arXiv:1803.07943 [hep-ph]].

[23] G. Apollinari, I. Béjar Alonso, O. Brüning, M. Lamont, and L. Rossi, "High-Luminosity Large Hadron Collider (HL-LHC): Preliminary Design Report”, CERN Yellow Reports: Monographs CERN-2015-005, doi:10.5170/CERN-2015-005 [https://cds.cern.ch/record/2116337]

[24] ATLAS Collaboration, "Studies on the impact of an extended Inner Detector tracker and a forward muon tagger on $W^{ \pm} W^{ \pm}$scattering in $p p$ collisions at the High-Luminosity LHC with the ATLAS experiment," ATL-PHYS-PUB-2017-023, [https://cds.cern.ch/record/2298958].

[25] CMS Collaboration, "Technical Proposal for the Phase-II Upgrade of the CMS Detector," CERN-LHCC-2015-010. LHCC-P-008. CMS-TDR-15-02, [https://cds.cern.ch/record/2020886]. 
[26] A. Ballestrero, E. Maina and G. Pelliccioli, " $W$ boson polarization in vector boson scattering at the LHC,” JHEP 1803 (2018) 170 doi:10.1007/JHEP03(2018)170 [arXiv:1710.09339 [hep-ph]]. 